\title{
VANISHING THEOREMS ON COMPLETE MANIFOLDS WITH WEIGHTED POINCARÉ INEQUALITY AND APPLICATIONS
}

\author{
HAI-PING FU AND DENG-YUN YANG
}

\begin{abstract}
Two vanishing theorems for harmonic map and $L^{2}$ harmonic 1-form on complete noncompact manifolds are proved under certain geometric assumptions, which generalize results of [13], [15], [18], [19], and [20]. As applications, we improve some main results in [2], [4], [6], [9], [12], [20], [22], [24], and [25].
\end{abstract}

\section{$\S 1$. Introduction}

Let $M^{n}$ be a minimal hypersurface in $\mathbb{R}^{n+1} . M$ is said to be stable if

$$
0 \leq \int_{M}\left(|\nabla \varphi|^{2}-|A|^{2} \varphi^{2}\right), \quad \forall \varphi \in C_{0}^{\infty}(M),
$$

where $|A|$ is the norm of the second fundamental form of $M$. For some number $0<\delta \leq 1$, it is defined that $M$ is $\delta$-stable if

$$
0 \leq \int_{M}\left(|\nabla \varphi|^{2}-\delta|A|^{2} \varphi^{2}\right), \quad \forall \varphi \in C_{0}^{\infty}(M) .
$$

Obviously, given $\delta_{1}>\delta_{2}, \delta_{1}$-stable implies $\delta_{2}$-stable. So, that $M$ is stable implies that $M$ is $\delta$-stable.

In [20], using harmonic map techniques, Schoen and Yau studied the fundamental group of a manifold of nonnegative Ricci curvature and of a stable minimal hypersurface immersed into nonpositively curved ambient space. Pigola, Rigoli, and Setti ([18], [19]) proved a Liouville-type theorem

Received December 10, 2010. Revised February 28, 2011. Accepted August 31, 2011. 2010 Mathematics Subject Classification. Primary 58E20; Secondary 53C42.

Fu's work was supported by the Mathematical Tianyuan Youth Foundation of National Natural Science Foundation of China grant 11026109, China Postdoctoral Science Foundation grant 20110491394, Jiangxi Province Natural Science Foundation of China grant 2010GZS0149, and Youth Science Foundation of Education Department of Jiangxi Province of China grant GJJ11044. 
for harmonic maps on complete manifolds with weighted Poincaré inequality which generalizes, in some respects, classical work due to Schoen and Yau [20].

On the other hand, Cheng and Zhou [5] proved that if $M$ is an $((n-2) / n)$ stable complete minimal hypersurface in $\mathbb{R}^{n+1}$ and has bounded norm of the second fundamental form, then $M$ must either have only one end or be a catenoid. This result for minimal hypersurfaces relies on the study of complete manifolds with weighted Poincaré inequality which is of independent interest. In [17], Li and Wang studied complete manifolds with satisfying property $\left(\mathcal{P}_{\rho}\right)$ and obtained many theorems on rigidity. Cheng and Zhou [5] generalized one result of [17]. Li and the first author in [10] recently refined the main results due to Cheng and Zhou [5].

In this paper, we study an $n$-dimensional complete noncompact Riemannian manifold with weighted Poincaré inequality. As applications, we study complete noncompact submanifolds. To state some results, we recall some notation and definitions.

Let $M$ be an $n$-dimensional complete oriented submanifold isometrically immersed in an $(n+p)$-dimensional Riemannian manifold $N^{n+p}$. Fix a point $x \in M$, and choose a local orthonormal frame $\left\{e_{1}, e_{2}, \ldots, e_{n+p}\right\}$ such that $\left\{e_{1}, e_{2}, \ldots, e_{n}\right\}$ are tangent fields. For each $\alpha, n+1 \leq \alpha \leq n+p$, define a linear map $A_{\alpha}: T_{x} M \rightarrow T_{x} M$ by

$$
\left\langle A_{\alpha} X, Y\right\rangle=\left\langle\widetilde{\nabla}_{X} Y, e_{\alpha}\right\rangle
$$

where $X, Y$ are tangent fields and where $\widetilde{\nabla}$ denotes the Riemannian connection on $N^{n+p}$. We denote by $H$ the mean curvature vector of $M$ :

$$
H=\frac{1}{n} \sum_{\alpha=n+1}^{n+p}\left(\operatorname{Tr} A_{\alpha}\right) e_{\alpha} .
$$

For each $\alpha, n+1 \leq \alpha \leq n+p$, define a linear map $\phi_{\alpha}: T_{x} M \rightarrow T_{x} M$ by

$$
\left\langle\phi_{\alpha} X, Y\right\rangle=\langle X, Y\rangle\left\langle H, e_{\alpha}\right\rangle-\left\langle A_{\alpha} X, Y\right\rangle
$$

and a bilinear map $\phi: T_{x} M \times T_{x} M \rightarrow T_{x} M^{\perp}$ by

$$
\phi(X, Y)=\sum_{\alpha=n+1}^{n+p}\left\langle\phi_{\alpha} X, Y\right\rangle e_{\alpha} .
$$


It is easy to see that the tensor $\phi$ is traceless. Denote by $A$ the second fundamental form of $M$. We have

$$
|A|^{2}=|\phi|^{2}+n|H|^{2} \text {. }
$$

For $N^{n+p}$, we say that the $(n-1)$ th Ricci curvature of $N$ satisfies $\operatorname{Ric}_{(n-1)}(N) \geq c$ if, for all points $x \in N$ and for all $n$-dimensional subspaces $V \subset T_{x}(N)$, the curvature tensor $R$ satisfies

$$
\sum_{i=1}^{n}\left\langle R\left(e_{i}, v\right) v, e_{i}\right\rangle \geq c, \quad v \in V
$$

where $\left\{e_{1}, \ldots, e_{n}\right\}$ is an orthonormal basis for $V$. Then $\operatorname{Ric}_{(n-1)}(N) \geq(n-$ 1) $c$ implies that $\operatorname{Ric}_{n}(N) \geq n c$.

Let $M$ be a complete Riemannian manifold, and let $q: M \rightarrow R$ be a differentiable function. Consider the elliptic operator $L=\Delta+q$ associated to the quadratic form

$$
(\varphi,-L \varphi)=-\int_{M} \varphi L \varphi=\int_{M}\left(|\nabla \varphi|^{2}-q \varphi^{2}\right), \quad \forall \varphi \in C_{0}^{\infty}(M) .
$$

Here $\Delta$ is the Laplacian, and $\nabla \varphi$ is the gradient of $\varphi$. The index of $L$ is defined to be the supremum, over compact domains of $M$, of the number of negative eigenvalues of $L$ with Dirichlet boundary condition. If $M$ is an $n$-dimensional constant mean curvature hypersurface in $N^{n+1}$ of constant curvature $c$ and if $q=n c+|A|^{2}$, then to say that $M$ is strongly stable is equivalent to saying that the index of $L$ is zero.

Let $H^{1}\left(L^{2}(M)\right)$ denote the space of $L^{2}$ harmonic 1 -forms on $M$. For convenience, throughout this article we assume that $\left(\delta-\sqrt{\delta^{2}-(n-2) \delta}\right) /$ $(n-1)<\beta<\left(\delta+\sqrt{\delta^{2}-(n-2) \delta}\right) /(n-1)$.

Now we can mention our results as follows.

THEOREM 1.1. Let $M$ be an $n$-dimensional complete noncompact Riemannian manifold, and let $W$ be a manifold of nonpositive sectional curvature. If the Ricci curvature of $M$ has the lower bound

$$
\operatorname{Ric}_{M}(x) \geq-(n-1) \tau(x), \quad x \in M
$$

where $\tau(x)$ satisfies Poincaré inequality

$$
\delta \int_{M} \tau \varphi^{2} \leq \int_{M}|\nabla \varphi|^{2}, \quad \forall \varphi \in C_{0}^{\infty}(M),
$$


where the constant $\delta$ is more than $(n-2)$, then any harmonic map $f: M \rightarrow$ $W$ is constant, provided that its energy density satisfies $\int_{B(R)}|d f|^{2 \beta}=o(R)$.

REMARK 1.2. The statement of Theorem 1.1 still holds when $\tau(x)$ satisfies Poincaré inequality

$$
\int_{M}\left(\frac{(n-1)^{2}}{n} \tau+\epsilon\right) \varphi^{2} \leq \int_{M}|\nabla \varphi|^{2}, \quad \forall \varphi \in C_{0}^{\infty}(M),
$$

for some $\epsilon>0$. Theorem 1.1 extends [20, Theorem 1], [18, Theorem 2.3], and [19, Theorem 6.1].

THEOREM 1.3. Let $M$ be an $n$-dimensional complete noncompact Riemannian manifold. If the Ricci curvature of $M$ has the lower bound

$$
\operatorname{Ric}_{M}(x) \geq-(n-1) \tau(x), \quad x \in M
$$

where $\tau(x)$ satisfies Poincaré inequality

$$
\delta \int_{M} \tau \varphi^{2} \leq \int_{M}|\nabla \varphi|^{2}, \quad \forall \varphi \in C_{0}^{\infty}(M),
$$

where the constant $\delta$ is more than $(n-1)^{2} / n$, then $H^{1}\left(L^{2}(M)\right)=0$.

REMARK 1.4. The statement of Theorem 1.3 still holds when $\tau(x)$ satisfies Poincaré inequality

$$
\int_{M}\left(\frac{(n-1)^{2}}{n} \tau+\epsilon\right) \varphi^{2} \leq \int_{M}|\nabla \varphi|^{2}, \quad \forall \varphi \in C_{0}^{\infty}(M),
$$

for some $\epsilon>0$. Theorem 1.3 is rewritten as follows.

THEOREM $1.3^{\prime}$. Let $M$ be an $n$-dimensional complete noncompact Riemannian manifold. For some $\epsilon>0, \forall x \in M$, if one of the following cases occurs:

(1) $\operatorname{Ric}_{M}(x) \geq-(n /(n-1)-\epsilon) \rho(x)$;

(2) $\operatorname{Ric}_{M}(x) \geq-(n /(n-1) \rho(x))+\epsilon$, where $\rho(x)$ satisfies Poincaré inequality

$$
\int_{M} \rho \varphi^{2} \leq \int_{M}|\nabla \varphi|^{2}, \quad \forall \varphi \in C_{0}^{\infty}(M) ;
$$

then $H^{1}\left(L^{2}(M)\right)=0$. 
So Theorem 1.3 can be regarded as generalizations of Lam [13, Theorems $0.5,3.4,3.5]$ since the $\rho$ in Lam's theorem satisfies some conditions (see [15, Theorem 4.2], [12, Theorem 1.1]).

As applications of Theorem 1.1 and 1.3, we obtain the following.

THEOREM 1.5. Let $M$ be an $n$-dimensional complete noncompact submanifold isometrically immersed in an $(n+p)$-dimensional manifold $N^{n+p}$ with $\operatorname{Ric}_{(n-1)}(N) \geq(n-1) c$. Assume that the index of the operator $\Delta+$ $\left(n c+|A|^{2}\right)$ is zero. If

$$
\left(\delta^{2}-4 n+4\right)|A|^{2} \leq 8 n(n-1)(n+\delta) c,
$$

where the constant $\delta$ is more than $(n-2)$, then any harmonic map from $M$ to a manifold with nonpositive curvature is constant, provided that its energy density satisfies $\int_{B(R)}|d f|^{2 \beta}=o(R)$. In particular, if $\delta$ is more than $(n-1)^{2} / n$, then $H^{1}\left(L^{2}(M)\right)=0$ and $M$ has at most one nonparabolic end.

Corollary 1.6. Let $M^{n}(n \leq 7)$ be an $n$-dimensional complete noncompact submanifold isometrically immersed in an $(n+p)$-dimensional manifold $N^{n+p}$ of nonnegative $(n-1)$ th Ricci curvature. If the index of the operator $\Delta+|A|^{2}$ is zero, then any harmonic map from $M$ to a manifold with nonpositive curvature is constant, provided that its energy density satisfies $\int_{B(R)}|d f|^{2 \beta}=o(R)$.

\section{§. Proof of main theorems}

Proof of Theorem 1.1. In [19, Proposition 1.3], Pigola, Rigoli, and Setti give a refined Kato inequality,

$$
|\nabla d f|^{2} \geq\left.\frac{n}{n-1}|\nabla| d f\right|^{2}
$$

Combining with (4), by the Bochner-type formula for harmonic maps between Riemannian manifolds (see [7]) and the nonpositivity of the sectional curvature of $W$, we have

$$
|d f| \Delta|d f| \geq \frac{1}{n-1}|\nabla| d f||^{2}+\operatorname{Ric}_{M}(d f, d f) .
$$


By using (5) and (2), we compute

$$
\begin{aligned}
|d f|^{\alpha} & \Delta|d f|^{\alpha} \\
& =|d f|^{\alpha}\left(\alpha(\alpha-1)|d f|^{\alpha-2}|\nabla| d f||^{2}+\alpha|d f|^{\alpha-1} \Delta|d f|\right) \\
& =\left.\left.\frac{\alpha-1}{\alpha}|\nabla| d f\right|^{\alpha}\right|^{2}+\alpha|d f|^{2 \alpha-2}|d f| \Delta|d f| \\
& \geq\left.\left.\frac{\alpha-1}{\alpha}|\nabla| d f\right|^{\alpha}\right|^{2}+\alpha|d f|^{2 \alpha-2}\left(\frac{1}{n-1}|\nabla| d f||^{2}-(n-1) \tau(x)|d f|^{2}\right) \\
& \geq\left.\left.\left(1-\frac{n-2}{(n-1) \alpha}\right)|\nabla| d f\right|^{\alpha}\right|^{2}-(n-1) \alpha \tau(x)|d f|^{2 \alpha},
\end{aligned}
$$

where $\alpha$ is a positive constant.

Let $q \geq 0$, and let $\phi \in C_{0}^{\infty}(M)$. Multiplying (6) by $|d f|^{2 q \alpha} \phi^{2}$ and integrating over $M$, we obtain

$$
\begin{aligned}
(1- & \left.\frac{n-2}{(n-1) \alpha}\right)\left.\left.\int_{M}|d f|^{2 q \alpha}|\nabla| d f\right|^{\alpha}\right|^{2} \phi^{2} \\
\leq & \int_{M}|d f|^{(2 q+1) \alpha} \Delta|d f|^{\alpha} \phi^{2}+(n-1) \alpha \int_{M} \tau(x)|d f|^{2(q+1) \alpha} \phi^{2} \\
= & (n-1) \alpha \int_{M} \tau(x)|d f|^{2(q+1) \alpha} \phi^{2}-\left.\left.(2 q+1) \int_{M}|d f|^{2 q \alpha}|\nabla| d f\right|^{\alpha}\right|^{2} \phi^{2} \\
& \quad-2 \int_{M}|d f|^{(2 q+1) \alpha} \phi\left\langle\nabla \phi, \nabla|d f|^{\alpha}\right\rangle,
\end{aligned}
$$

which gives

$$
\begin{gathered}
\left.\left.\left(2(q+1)-\frac{n-2}{(n-1) \alpha}\right) \int_{M}|d f|^{2 q \alpha}|\nabla| d f\right|^{\alpha}\right|^{2} \phi^{2} \\
\leq(n-1) \alpha \int_{M} \tau(x)|d f|^{2(q+1) \alpha} \phi^{2} \\
\quad-2 \int_{M}|d f|^{(2 q+1) \alpha} \phi\left\langle\nabla \phi, \nabla|d f|^{\alpha}\right\rangle .
\end{gathered}
$$

Using the Cauchy-Schwarz inequality, we can rewrite (7) as

$$
\begin{aligned}
& \left.\left.\left(2(q+1)-\frac{n-2}{(n-1) \alpha}-\epsilon\right) \int_{M}|d f|^{2 q \alpha} \phi^{2}|\nabla| d f\right|^{\alpha}\right|^{2} \\
& \quad \leq \frac{1}{\epsilon} \int_{M}|d f|^{2(q+1) \alpha}|\nabla \phi|^{2}+(n-1) \alpha \int_{M} \tau(x)|d f|^{2(q+1) \alpha} \phi^{2} .
\end{aligned}
$$


On the other hand, replacing $\varphi$ by $|d f|^{(1+q) \alpha} \phi$ in inequality (3), we have

$$
\begin{aligned}
& \delta \int_{M} \tau(x)|d f|^{2(1+q) \alpha} \phi^{2} \\
& \leq\left.\left.(1+q)^{2} \int_{M}|d f|^{2 q \alpha}|\nabla| d f\right|^{\alpha}\right|^{2} \phi^{2}+\int_{M}|d f|^{2(1+q) \alpha}|\nabla \phi|^{2} \\
& \quad+2(1+q) \int_{M}|d f|^{(2 q+1) \alpha} \phi\left\langle\nabla \phi, \nabla|d f|^{\alpha}\right\rangle
\end{aligned}
$$

which gives

$$
\begin{aligned}
\delta \int_{M} \tau(x)|d f|^{2(1+q) \alpha} \phi^{2} \leq & \left.\left.(1+q)(1+q+\epsilon) \int_{M}|d f|^{2 q \alpha}|\nabla| d f\right|^{\alpha}\right|^{2} \phi^{2} \\
& +\left(1+\frac{1+q}{\epsilon}\right) \int_{M}|d f|^{2(1+q) \alpha}|\nabla \phi|^{2}
\end{aligned}
$$

If $2(q+1)-(n-2) /((n-1) \alpha)-\epsilon>0$, then by introducing (10) to (8), we obtain

$$
\left.\left.B \int_{M}|d f|^{2 q \alpha}|\nabla| d f\right|^{\alpha}\right|^{2} \phi^{2} \leq D \int_{M}|d f|^{2(1+q) \alpha}|\nabla \phi|^{2}
$$

where

$$
\begin{aligned}
B & =\left(2(q+1)-\frac{n-2}{(n-1) \alpha}-\epsilon\right) \delta-(n-1)(1+q)(1+q+\epsilon) \alpha \\
D & =\frac{\delta}{\epsilon}+\frac{(n-1) \alpha(1+q+\epsilon)}{\epsilon}
\end{aligned}
$$

Let $(1+q) \alpha=\beta ;$ thus, for $\left(\delta-\sqrt{\delta^{2}-(n-2) \delta}\right) /(n-1)<\beta<$ $\left(\delta+\sqrt{\delta^{2}-(n-2) \delta}\right) /(n-1)$, it is easy to see that $2(q+1)-(n-2) /$ $((n-1) \alpha)>0$ and that $(2(q+1)-(n-2) /((n-1) \alpha)) \delta-(n-1)(1+q)^{2} \alpha>$ 0 . Then we can choose $\epsilon>0$ sufficiently small so that $2(q+1)-(n-2) /$ $((n-1) \alpha)-\epsilon>0$ and $B>0$. It follows from (11) that the following inequality holds:

$$
\left.\left.\int_{M}|d f|^{2 q \alpha}|\nabla| d f\right|^{\alpha}\right|^{2} \phi^{2} \leq C \int_{M}|d f|^{2 \beta}|\nabla \phi|^{2},
$$

where $C$ is a constant that depends on $\delta, \alpha, \epsilon$, and $q$. Let $\phi$ be a smooth function on $[0, \infty)$ such that $\phi \geq 0, \phi=1$ on $[0, R]$ and $\phi=0$ in $[2 R, \infty)$ with 
$\left|\phi^{\prime}\right| \leq 2 / R$. Then considering $\phi \circ r$, where $r$ is the function in the definition of $B(R)$, we have from (12)

$$
\left.\left.\int_{M}|d f|^{2 q \alpha}|\nabla| d f\right|^{\alpha}\right|^{2} \phi^{2} \leq \frac{4 C}{R^{2}} \int_{B(2 R) \backslash B(R)}|d f|^{2 \beta} .
$$

Let $R \rightarrow+\infty$, by assumption that $\int_{B(R)}|d f|^{2 \beta}=o(R)$. From (13) we conclude that $\nabla|d f|^{\alpha}=0$ and that $|d f|$ is constant. Thus, if $|d f| \neq 0$, from (6) we get $\tau(x) \geq 0$. It follows by substituting the above $|d f|$ into (9) that

$$
\delta \int_{B(R)} \tau|d f|^{2 \beta} \leq \delta \int_{M} \tau|d f|^{2 \beta} \phi^{2} \leq \frac{1}{R^{2}} \int_{B(2 R)(p)}|d f|^{2 \beta} .
$$

So we conclude by letting $R \rightarrow+\infty$ that $\tau(x) \equiv 0$. Thus, by (2), we have $\operatorname{Ric}_{M}(x) \geq 0$. By [21, Theorem 4.1], we get that the volume of $B(R)$ satisfies $\operatorname{vol}(B(R)) \geq C(n) R$, and $\int_{B(R)}|d f|^{2 \beta} \geq C(n)|d f|^{2 \beta} R$. This is a contradiction since $\int_{B(R)}|d f|^{2 \beta}=o(R)$. Consequently, we have $|d f|=0$ and $d f=0$. This completes the proof of Theorem 1.1.

Proof of Theorem 1.3. For each $\omega \in H^{1}\left(L^{2}(M)\right)$, we have the following well-known Bochner formula:

$$
\Delta|\omega|^{2}=2\left(|\nabla \omega|^{2}+\operatorname{Ric}_{M}(\omega, \omega)\right)
$$

On the other hand, we have

$$
\Delta|\omega|^{2}=2\left(|\omega| \Delta|\omega|+|\nabla| \omega||^{2}\right) .
$$

From (14), (15), and the generalized version of Kato's inequality $n /$ $\left.(n-1)|\nabla| \omega\right|^{2} \leq|\nabla \omega|^{2}$ (see [16]), we obtain

$$
|\omega| \Delta|\omega| \geq \operatorname{Ric}_{M}(\omega, \omega)+\frac{1}{n-1}|\nabla| \omega||^{2}
$$

Combining with (2), we have

$$
|\omega| \Delta|\omega| \geq \frac{1}{n-1}|\nabla| \omega||^{2}-(n-1) \tau|\omega|^{2} .
$$

Using the same argument as Theorem 1.1, one can conclude that $H^{1}\left(L^{2}(M)\right)=0$. 


\section{§3. Application to submanifolds}

Before proving our results, we list some known facts that we need.

Definition 3.1. Let $D \subset M$ be a compact subset of $M$. An end $E$ of $M$ with respect to $D$ is a connected unbounded component of $M \backslash D$. When we say that $E$ is an end, it is implicitly assumed that $E$ is an end with respect to some compact subset $D \subset M$.

Definition 3.2. A manifold is said to be parabolic if it does not admit a positive Green's function. Conversely, a nonparabolic manifold is one which admits a positive Green's function. An end $E$ of a manifold is said to be $n o n-$ parabolic if it admits a positive Green's function with Neumann boundary condition on $\partial E$. Otherwise, it is said to be parabolic.

Lemma 3.3 ([14, Theorem 2.1]). Let $M$ be a complete manifold. Let $\mathcal{H}_{D}^{0}(M)$ denote the space of bounded harmonic functions with finite Dirichlet integral. Then the number of nonparabolic ends of $M$ is at most the dimension of $\mathcal{H}_{D}^{0}(M)$.

Lemma 3.4 ([1, Section 2], [3, p. 22], [16, Corollary 4]). Let E be an end of a complete manifold. Suppose that for some $\nu \geq 1, E$ satisfies a Sobolev-type inequality of the form

$$
\left(\int_{E}|f|^{2 \nu}\right)^{\frac{1}{\nu}} \leq C \int_{E}|\nabla f|^{2}, \quad \forall f \in C_{0}^{1}(E) .
$$

Then (1) if $\nu=1$, then $E$ must either have finite volume or be nonparabolic; (2) if $\nu>1$, then $E$ must be nonparabolic.

THEOREM 3.5. Let $M$ be an n-dimensional complete noncompact submanifold isometrically immersed in an $(n+p)$-dimensional Riemannian manifold $N^{n+p}$ with $\operatorname{Ric}_{(n-1)}(N) \geq(n-1) c$. Assume that the index of the operator $\Delta+\left(n c+|A|^{2}\right)$ is zero. If

$$
0 \leq\left[\sqrt{n-1}(n-\delta)|\phi|^{2}-(n-2) \sqrt{n} \delta|\phi||H|+n \sqrt{n-1}(n+\delta)\left(|H|^{2}+c\right)\right]
$$

where $\delta$ is more than $(n-2)$, then any harmonic map from $M$ to a manifold with nonpositive curvature is constant, provided that its energy density satisfies $\int_{B(R)}|d f|^{2 \beta}=o(R)$. In particular, if $\delta$ is more than $(n-1)^{2} / n$, then $H^{1}\left(L^{2}(M)\right)=0$ and $M$ has at most one nonparabolic end. 
Proof. Shiohama and $\mathrm{Xu}$ [23] proved that the following estimate holds for Ricci curvature of a submanifold $M$ in a Riemannian manifold $N^{n+p}$

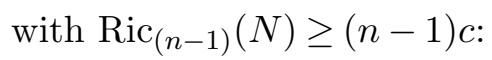

$$
\operatorname{Ric}_{M} \geq \frac{n-1}{n}\left(n c+2 n|H|^{2}-\frac{n(n-2)}{\sqrt{n(n-1)}}|H| \sqrt{|A|^{2}-n|H|^{2}}-|A|^{2}\right) .
$$

Applying the above inequality to the traceless second fundamental form $|\phi|$ and using the identity $|A|^{2}=|\phi|^{2}+n|H|^{2}$, we get

$$
\begin{aligned}
\operatorname{Ric}_{M} \geq & (n-1) c+(n-1)|H|^{2} \\
& -\frac{(n-2) \sqrt{n(n-1)}|\phi||H|}{n}-\frac{(n-1)|\phi|^{2}}{n} .
\end{aligned}
$$

Let us choose $\tau=|\phi|^{2} / n+((n-2)|\phi||H|) / \sqrt{n(n-1)}-c-|H|^{2}$ in Theorem 1.1; thus, $\operatorname{Ric}_{M}(x) \geq-(n-1) \tau(x)$.

On the other hand,

$$
\begin{aligned}
\int_{M}\left(n c+|A|^{2}-\delta \tau\right) \varphi^{2} & \\
= & \int_{M}\left((n+\delta)\left(c+|H|^{2}\right)+\frac{n-\delta}{n}|\phi|^{2}\right) \varphi^{2}-\int_{M} \frac{(n-2) \delta|\phi||H|}{\sqrt{n(n-1)}} \varphi^{2} \\
= & \int_{M}\left(\left(\sqrt{n-1}(n-\delta)|\phi|^{2}-(n-2) \sqrt{n} \delta|\phi||H|\right.\right. \\
& \left.\left.\quad+n \sqrt{n-1}(n+\delta)\left(|H|^{2}+c\right)\right) /(n \sqrt{n-1})\right) \varphi^{2}
\end{aligned}
$$

By assumption, from the above inequality we obtain

$$
\delta \int_{M} \tau \varphi^{2} \leq \int_{M}\left(n c+|A|^{2}\right) \varphi^{2} \leq \int_{M}|\nabla \varphi|^{2} .
$$

Hence, by Theorems 1.1 and 1.3 and Lemma 3.3, we complete the proof of Theorem 3.5.

By Theorem 3.5, and using the Schwarz inequality, we get the following.

Corollary 3.6. Let $M$ be an $n$-dimensional complete noncompact submanifold isometrically immersed in an $(n+p)$-dimensional Riemannian manifold $N^{n+p}$ with $\operatorname{Ric}_{(n-1)}(N) \geq(n-1) c$. Assume that the index of the operator $\Delta+\left(n c+|A|^{2}\right)$ is zero. If one of the following cases occurs: 
(1) $n^{2}\left(\delta^{2}-4 n+4\right)|H|^{2} \leq 4(n-1)\left(n^{2}-\delta^{2}\right) c$;

(2) $n\left(\delta^{2}-4 n+4\right)|\phi|^{2} \leq 4(n-1)(n+\delta)^{2} c$;

where $\delta$ is more than $(n-2)$, then any harmonic map from $M$ to a manifold with nonpositive curvature is constant, provided that its energy density satisfies $\int_{B(R)}|d f|^{2 \beta}=o(R)$. In particular, if $\delta$ is more than $(n-1)^{2} / n$, then $H^{1}\left(L^{2}(M)\right)=0$ and $M$ has at most one nonparabolic end.

Corollary 3.7. Let $M^{n}$ be an n-dimensional complete noncompact minimal submanifold isometrically immersed in an $(n+p)$-dimensional manifold $N^{n+p}$ of nonnegative $(n-1)$ th Ricci curvature. If the index of the operator $\Delta+|A|^{2}$ is zero, then any harmonic map with finite energy from $M$ to a manifold with nonpositive curvature is constant and $H^{1}\left(L^{2}(M)\right)=0$. Moreover, $M$ has at most one nonparabolic end.

REMARK 3.8. When $M$ is an $n$-dimensional constant mean curvature hypersurface in $N^{n+1}$ with $\operatorname{Ric}_{(n-1)}(N) \geq(n-1) c$, that $M$ is strongly stable implies that the index of the operator $\Delta+\left(n c+|A|^{2}\right)$ is zero. Thus, if $M^{n}$ in Corollaries 3.6 and 3.7 is a complete strongly stable hypersurface with constant mean curvature in $N^{n+1}$ with $\operatorname{Ric}_{(n-1)}(N) \geq(n-1) c$, then the statements of Corollaries 3.6 and 3.7 are also true. In particular, when $N^{n+1}$ is a space form, by Lemma $3.4, M$ has only one end. So Corollaries 3.6 and 3.7 can be considered as generalizations of some main results in [4], [9], [12], and [20].

It is easy to see that we get Theorem 1.5 by Corollary 3.6. By Theorems 1.1 and 1.3, we have the following Corollaries 3.9 and 3.11 by using (18) and Lemmas 3.3 and 3.4 .

Corollary 3.9. Let $M$ be an $n$-dimensional complete noncompact minimal submanifold isometrically immersed in $\mathbb{H}^{n+p}$. If the index of the operator $\Delta+\left(-n+|A|^{2}\right)$ is zero and if

$$
\lambda_{1}(M)>\frac{2 n(n-1)^{2}}{2 n-1},
$$

then any harmonic map with finite energy from $M$ to a manifold with nonpositive curvature is constant and $H^{1}\left(L^{2}(M)\right)=0$. Moreover, $M$ has only one end.

REMARK 3.10. Corollary 3.9 is better than the main theorems in [22] and $[11]$. 
Corollary 3.11. Let $M$ be an $n$-dimensional complete noncompact minimal submanifold isometrically immersed in an $(n+p)$-dimensional Riemannian manifold $N^{n+p}$ of nonnegative $(n-1)$ th Ricci curvature. If the index of the operator $\Delta+\delta|A|^{2}$ is zero, where $\delta$ is more than $(n-1)^{2} / n^{2}$, then $H^{1}\left(L^{2}(M)\right)=0$. Moreover, $M$ has only one end.

REMARK 3.12. Corollary 3.11 can be regarded as generalizations of $[2$, Theorem 1], [24, Theorem 1.2], and [8, Theorem 1.1].

REMARK 3.13. Based on Theorems 1.1 and 1.3, by using the same arguments as before, we can improve the main results of [6], [24], and [25].

Acknowledgment. The authors would like to thank the referees for some helpful suggestions.

\section{REFERENCES}

[1] K. Akutagawa, Yamabe metrics of positive scalar curvature and conformally flat manifolds, Differential Geom. Appl. 4 (1994), 239-258.

[2] H. D. Cao, Y. Shen, and S. Zhu, The structure of stable minimal hypersurfaces in $R^{n+1}$, Math. Res. Lett. 4 (1997), 637-644.

[3] G. Carron, $L^{2}$ harmonic forms on noncompact manifolds, preprint, arXiv:0704.3194 [math.DG]

[4] X. Cheng, L. F. Cheung, and D. T. Zhou, The structure of weakly stable constant mean curvature hypersurfaces, Tohoku Math. J. (2) 60 (2008), 101-121.

[5] X. Cheng and D. T. Zhou, Manifolds with weighted Poincaré inequality and uniqueness of minimal hypersurfaces, Comm. Anal. Geom. 17 (2009), 139-154.

[6] M. P. do Carmo, Q. L. Wang, and C. Y. Xia, Complete submanifolds with bounded mean curvature in a Hadamard manifold, J. Geom. Phys. 60 (2010), 142-154.

[7] J. Eells and J. H. Sampson, Harmonic mappings of Riemannian manifolds, Amer. J. Math. 86 (1964), 109-160.

[8] H. P. Fu, The structure of $\delta$-stable minimal hypersurface in $\mathbb{R}^{n+1}$, Hokkaido Math. J. 40 (2011), 103-110.

[9] H. P. Fu and Z. Q. Li, On stable constant mean curvature hypersurfaces, Tohoku Math. J. (2) 62 (2010), 383-392.

[10] - The structure of complete manifolds with weighted Poincaré inequality and minimal hypersurfaces, Internat. J. Math. 21 (2010), 1421-1428.

[11] H. P. Fu and H. W. Xu, Weakly stable constant mean curvature hypersurfaces, Appl. Math. J. Chinese Univ. Ser. B 24 (2009), 119-126.

[12] - Vanishing results on complete manifolds with Poincaré inequality and applications, preprint, 2009.

[13] K. H. Lam, Results on weighted Poincaré inequality of complete manifolds, Trans. Amer. Math. Soc. 362 (2010), 5043-5062.

[14] P. Li and L. F. Tam, Harmonic functions and the structure of complete manifolds, J. Differential Geom. 35 (1992), 359-383.

[15] P. Li and J. P. Wang, Complete manifolds with positive spectrum, J. Differential Geom. 58 (2001), 501-534. 
[16] - Minimal hypersurfaces with finite index, Math. Res. Lett. 9 (2002), 95-103.

[17] Weighted Poincaré inequality and rigidity of complete manifolds, Ann. Sci. Éc. Norm. Supér. (4) 39 (2006), 921-982.

[18] S. Pigola, M. Rigoli, and A. G. Setti, Vanishing theorems on Riemannian manifolds, and geometric applications, J. Funct. Anal. 229 (2005), 424-461.

[19] - Vanishing and Finiteness Results in Geometric Analysis: A Generalization of the Bochner Technique, Progr. Math. 266, Birkhäuser Basel, 2008.

[20] R. Schoen and S. T. Yau, Harmonic maps and the topology of stable hypersurfaces and manifolds with non-negative Ricci curvature, Comment. Math. Helv. 51 (1976), 333-341.

[21] , "Lectures on differential geometry" in Conference Proceedings and Lecture Notes in Geometry and Topology, I, International Press, Cambridge, 1994.

[22] K. Seo, $L^{2}$ harmonic 1-forms on minimal submanifolds in hyperbolic space, J. Math. Anal. Appl. 371 (2010), 546-551.

[23] K. Shiohama and H. W. Xu, The topological sphere theorem for complete submanifolds, Compos. Math. 107 (1997), 221-232.

[24] Q. L. Wang, Complete submanifolds in manifolds of partially non-negative curvature, Ann. Global Anal. Geom. 37 (2010), 113-124.

[25] Q. L. Wang and C. Y. Xia, Complete submanifolds of manifolds of negative curvature, Ann. Global Anal. Geom. 39 (2011), 83-97.

Hai-Ping Fu

Department of Mathematics

Nanjing University

Nanjing 210093

People's Republic of China

and

Department of Mathematics

Nanchang University

Nanchang 330031

People's Republic of China

mathfu@126.com

Deng-Yun Yang

College of Mathematics and Information Science

Jiangxi Normal University

Nanchang 330022

People's Republic of China

yangdengyun $@ 126 . c o m$ 